\title{
Pupillometry system for use in social psychology
}

\author{
PAUL C. BERNHARDT, JAMES M. DABBS, JR., and JASMIN K. RIAD \\ Georgia State University, Atlanta, Georgia
}

\begin{abstract}
It is now relatively easy to implement pupillometry in social psychological research. We describe a system in which a Macintosh computer presents visual and auditory stimuli to subjects while monitoring an infrared pupillometer. The computer records information on subject, task, and pupil diameter into a data file for input into standard statistical analysis programs. Pupil diameter is measured continuously while subjects respond to stimuli that last for several seconds or minutes. A demonstration experiment verifies the usefulness of the system. The ability of the Macintosh computer to easily handle visual and auditory stimuli and to record responses lasting a considerable length of time makes the system well suited for studying pupil responses to complex social stimuli. This system is also well suited for the researcher initiating a research program using pupillometry. Ease of operation makes using a large number of subjects feasible.
\end{abstract}

The eyes are a window to the soul, or at least to the mind, and for decades psychologists have looked to the eyes for clues to mental processes. Pupil responses provide indicators of cognitive processing, attention, and brain activation (Beatty, 1982; Beatty \& Wagoner, 1978). In the past, measuring pupil responses was tedious and required considerable technical sophistication. We believe our system makes it somewhat easier for a new researcher to implement pupillometry in studying the cognitive underpinnings of social psychological processes. The system records data while simultaneously presenting complex stimuli. The present paper describes the system and presents preliminary data to indicate its usefulness.

Pupil dilation is generally regarded as a measure of brain activation, indicating the intensity of concentration or interest in a stimulus (Tryon, 1975). Initial task-evoked pupillary responses occur quite quickly (300-500 msec) and dissipate equally quickly when processing ceases (Beatty, 1986). This brief time interval is appropriate for some processes in social psychology, such as affect and impression formation. However, other phenomena of interest in social psychology involve prolonged reactions to complex stimuli. For the longer term responses, methods of data collection and analysis are needed that preserve the richness of the phenomena. Currently available computers make statistical analysis of patterns of change over time a practical reality, though in order to conduct these analyses, the continuous nature of the data must be preserved. Our system

P. C. B. is now pursuing a doctoral program at the Department of Psychology, University of Utah. This research was supported in part by a National Science Foundation grant (BNS-9021393) and a Georgia State University Chancellor's Initiative grant to J.M.D. Portions of this research were presented at the annual meeting of the American Psychological Association, Washington, DC, August 1992. We thank R. Tipton for his valuable suggestions during the development of these computer programs and $M$. Dabbs for her help in adjusting the color of the Bellows paintings. Correspondence should be addressed to J. M. Dabbs, Department of Psychology, Georgia State University, University Plaza, Atlanta, GA 30303 (e-mail: psyjmd@gsusgi2.gsu.edu). records a continuous stream of pupil diameter scores in a format that can be easily transferred to a mainframe computer for analysis.

The system we have developed directly displays complex stimuli while recording pupil diameters and is adaptable to a variety of experimental procedures. A single computer replaces the more common arrangement in which two linked computers perform the separate tasks of presenting stimuli and recording data, or an arrangement in which a computer controls some external device that presents stimuli. Using a single computer gives the advantage of greater reliability and ease of setup because of fewer components. The actions of presenting stimuli and recording data proceed without interference from each other.

Several systems have been developed that control stimuli while collecting pupillary response data. McConkie, Scouten, Bryant, and Wilson (1988) developed a system for IBM-compatible computers that uses a combination of C language and assembly language routines for pupillometry. Programming in assembly language requires greater sophistication than programming in higher level languages such as Pascal. Blumenthal and Cooper (1990) developed a system for Macintosh computers that uses Pascal programs to measure the startle reflex eye blink. However, in Blumenthal and Cooper's system, as well as McConkie et al.'s system, the computer both gathers the data and controls external equipment to present stimuli. The control of external equipment requires considerably more technical sophistication than does a system such as ours, for which the stimuli are presented on the computer's display screen.

The difficulty of control of external equipment is addressed by our system. Compatibility between external devices and the computer controlling the experiment has been a major barrier to implementation in previous pupillometry systems. Technicians must be knowledgeable about many devices that use different methods of coding information. Any device that displays stimuli must be controlled in some manner to ensure consistent experimental conditions. Typically an output interface card in the computer sends sig- 
nals to the internal control mechanisms of the stimulus display device. Transformation of the signals to ensure compatibility, construction of suitable signal transmission cable, and testing to eliminate errors in the system are all problems requiring the attention of a technician who is well versed in these pieces of equipment and procedures. Using the intrinsic capabilities of the Macintosh computer system, as described in this paper, means that compatibility between the computer and the stimulus presentation device has already been addressed by the manufacturer. Since less technical sophistication is required for implementation, we believe our system offers an advantage to researchers who want to initiate a new research program using pupillometry.

Other systems have been developed that monitor the eye while simultaneously displaying video information. Hutchinson and his colleagues have described an IBMcompatible system that uses direction of gaze information to execute commands displayed on the computer screen (Frey, White, \& Hutchinson, 1990; Hutchinson, White, Martin, Reichert, \& Frey, 1989). The system detects the user's gaze dwelling on a given command, which is then executed. Hutchinson's system is intended for use as a computer interface for the physically impaired. Although it might be adapted for psychophysiological measures, such adaptation has not yet been presented.

Our system has two principal components. The first is the infrared pupillometer. It is connected to the second element, which is the Macintosh computer system. A computer program controls the Macintosh and organizes the data. The following describes the system in greater detail.

\section{SYSTEM DESCRIPTION}

The system has two major hardware subsystems (See Figure 1). A corneal reflection tracking device, the ISCAN Model RK-426 (ISCAN, Cambridge, MA) is used as a pupillometer. An infrared sensitive television camera

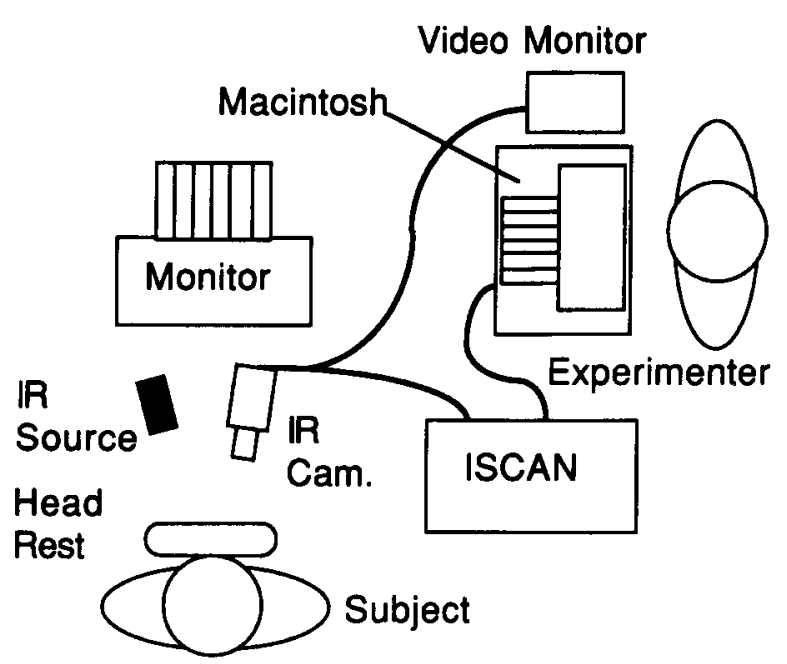

Figure 1. Schematic layout of equipment. transmits an image of the infrared illuminated eye to the ISCAN. The pupil absorbs infrared and appears as a dark spot to the ISCAN equipment. The ISCAN equipment uses contrast and reflection detection algorithms to compute pupil diameter and direction of gaze. This information is continuously outputted in both analog voltage and digital formats.

The second subsystem is a Macintosh computer (model IIci) with a high-resolution color video display and a McAdios Jr. analog-to-digital (A/D) interface card (GW Instruments, Sommerville, MA). A Pascal program ${ }^{1}$ controls the presentation of stimuli to subjects either aurally through headphones or loudspeakers, or visually through a color video display. The program instructs the $A / D$ card to interrupt the operation of the program at regular intervals to read and store pupil measurements.

The program keeps track of information regarding the subject and the subject's tasks. By using an internal clock, it synchronizes the pupillometry record with critical timing indexes and task labels. At the end of a session the program constructs a data file that includes the following on each line of data: subject number, task type, task within type, and line number (representing time) within task. Each line contains a new pupil diameter value; therefore, the data file represents repeated measurements of pupil diameter. Storing the data in this format facilitates later statistical analysis.

The program, written exclusively in compiled Pascal (Think Pascal, Symantec, Cupertino, CA), utilizes several features unique to the Macintosh operating system. The sound and picture stimuli are stored in "resource" files accessed by the program. (A resource file is a special class of data structure used in Macintosh computers that separates data used by the program from the main application program itself.) Storing the stimuli in this manner makes it possible to change the stimulus set without modifying the program code or, in many cases, recompilation. Most Macintosh applications used for sound and picture editing have the ability to create resource files. A particular program, ResEdit (Apple Computer, Cupertino, CA), allows for easy editing of resource files.

The program displays pictures in 24-bit color in a standard Macintosh window. The Macintosh operating system allows the display of differently sized pictures against a medium gray background. The picture being displayed carries display size information within its file. This is used to size the window for display of the picture so the background is automatically reconfigured for each picture as necessary.

This versatility minimizes problems of background brightness influencing pupil diameter. Each picture may be of different size. Medium gray can be used as a background color on the Macintosh. The automatic sizing of the window means that the background will automatically be filled with this neutral color. This consistency in background means that pupil diameter changes can be attributed to stimulus differences rather than brightness differences.

The Macintosh is particularly well suited for presentation of sounds. Built into the system software and hardware is the capability of sampling sounds at $22 \mathrm{kHz}$ for digital 
encoding. Application programs can be used to manipulate sound data to introduce a variety of differences between sounds that may affect pupil diameter. These same programs can store sounds as resources similar to pictures. Versions of the operating program for our system have used sounds as stimuli (Bernhardt, Cummings, Dabbs, Riad, Hopper, \& Clay, 1992).

\section{SYSTEM CAPABILITIES}

The system has demonstrated useful capabilities. The computer data collection rate greatly exceeds the maximum sampling rate of the ISCAN equipment. Visual and auditory stimuli can be presented at a rate consistent with the social phenomenon of interest. The system is user friendly; each subject takes only about 15 min to run, including instructions and debriefing. The following describes system capabilities and limitations in greater detail.

The MacAdios, Jr., analog-to-digital card is not a limiting component in gathering data. It can record samples as fast as every $100 \mathrm{msec}$ in this experimental setup. An advantage is its use of background interrupts to gather data. After being initialized by the program, it temporarily pauses the execution of the program at commanded intervals to get pupil diameter value from the ISCAN machine. After the data are stored, the program resumes. This operation is rapid, so there is no degradation or delay in video presentation.

The ISCAN pupillometer is the most conspicuous limiting factor in data collection. It produces a new measurement of pupil diameter every $1 / 60 \mathrm{sec}$. Therefore, data gathering rates that exceed $60 / \mathrm{sec}$ are redundant.

The system allows for versatility in displaying stimuli while pupillometry data are being recorded. The timing is handled by delay loops that are adjusted for different experiments. The delay loops keep the timing of stimuli display to within $1 / 60$ sec from task to task. With typical stimuli presentation times of 5-15 sec, error of less than $1 / 60 \mathrm{sec}$ in display timing is negligible. Commands recently developed for the Macintosh by the manufacturer (TimeManager commands) are presently being incorporated, and they should reduce timing error to about $20 \mathrm{msec}$.

Any computer takes a finite amount of time to display a given image. In the current version of the program, the full screen picture typically takes about $1 / 4 \mathrm{sec}$ to display. Pupil diameters recorded during this time are removed from the analysis. Off-screen drawing methods are presently being incorporated that will reduce the image display time to the refresh rate of the monitor (about $1 / 60 \mathrm{sec}$ ).

The presentation of sounds as stimuli is easily accomplished. A digital sound file on the Macintosh's disk drive carries precise timing information that ensures consistent playback time, frequency, and timbre. Once initialized, a short and consistent delay (heard as silence) occurs before the sound actually starts. It is possible through the intrinsic stereo hardware of the Macintosh to play sounds in either or both ears selectively. As in video presentation, the interruption of the program to record a pupil diameter causes no degradation in sound quality.

\section{DEMONSTRATION STUDY}

This section describes a study that demonstrates the potential of the system in social psychological experiments. The study examined the interest of male and female subjects viewing violent and peaceful scenes. We expected to find an interaction between the gender of subject and violence of pictures in the pattern (over time) of pupil diameter.

\section{Method}

Eighteen male and 21 female undergraduates participated to fulfill an introductory psychology course requirement. Subjects sat in a comfortable chair and rested their foreheads and chins against a headrest to fix the position of an eye for the camera and infrared light source. They were asked to pay attention to what they saw. Visual stimull were presented on a $30-\mathrm{cm}$ (diagonal) color monitor positioned about $70 \mathrm{~cm}$ from the subject.

We examıned pupil responses while subjects viewed paintings by George Bellows. High-quality reproductions of the paintıngs were scanned into the computer and presented to subjects as 24-bit color images. Five boxing scenes and five peaceful scenes of seascapes and people engaged in commonplace activities were presented to each subject, with the fight/peaceful order counterbalanced across subjects. Each picture was presented for $6 \mathrm{sec}$, preceded by $6 \mathrm{sec}$ of a mosaic made up of the patterns and colors shown in the following picture. The intention was for the mosaic to provide a baseline for each picture by virtue of the image being composed of the same intensity of colors. Pupil size was recorded every $1 / 4 \mathrm{sec}$. Salivary testosterone levels were also measured for 14 male subjects in this study using the methods similar to those described by Dabbs (1990, 1991), because of a widely reported link between testosterone and aggression (Archer, 1991).

\section{Results}

Data were analyzed using SAS (SAS Institute, 1982). As an initial step in data processing, pupil diameter scores were sorted into a frequency distribution for each subject. Scores in the tails of the distribution, beyond the point where the frequency dropped at least once to zero, were identified as outliers. Since these outlier scores seemed to represent data associated with eye blinking, each outlier was replaced by the last valid score preceding it in the subject's original stream of data. A small number of scores (representing $2.03 \%$ ) were replaced in that manner.

Examination of plots of pupil diameter across the entire experimental session suggested a problem with using mosaics as baselines. The mosaics were quite striking in appearance, and subjects dilated more in response to them than to the pictures. The first $2 \mathrm{sec}$ of each picture was discarded because subjects appeared primarily to be recovering from dilation to the preceding mosaic. Dilation at the 2 -sec point was taken as a baseline for each picture, and all pupil scores were converted to percent of baseline scores.

An initial gender $(2) \times$ time $(16) \times$ picture $(10)$ repeated measures analysis of variance (ANOVA) revealed different patterns of change across the 10 pictures, with effects of time $[F(14,476)=3.02, p<.001, \varepsilon=.37]$, picture $[F(9,306)=2.70, p<.01, \varepsilon=.82]$, and time $\times$ picture $[F(126,4284)=1.64, p<.001, \varepsilon=.23] .{ }^{2}$ When the 10 pictures were grouped into two content categories (fight and peaceful), a repeated measure ANOVA showed effects of time $[F(14,476)=3.02, p<.001, \varepsilon=.37]$ and 
of time $\times$ content $\times \operatorname{sex}[F(14,476)=1.79, p<.05, \varepsilon=$ $.44]$. Figure 2 shows the three-way interaction across time. Females dilated more to peaceful pictures. Males dilated more to fight pictures.

A median split of testosterone scores was used to divide male subjects into two groups. We expected hightestosterone males to dilate more to fight than to peaceful pictures. However, this was not the case. In a 2 (testosterone) $\times 2$ (content) $\times 16$ (time) repeated measures ANOVA, there was a significant testosterone $\times$ time effect $[F(14,182)=1.89, p<.05, \varepsilon=.29]$. As indicated in Figure 3 , high-testosterone males dilated more over time to both types of pictures than did low-testosterone males.

\section{Discussion}

The system produced consistent and analyzable results in this demonstration. We were able to discern dilation differences between males and females and differences between high- and low-testosterone males. We also detected effects that were not apparent in a casual inspection of the raw data. With appropriate data analysis, this system can detect changes in slope that are subtle but may prove meaningful when appropriate controls and manipulation checks are in place.

We were also able to demonstrate the ease of operation of the system. No subjects in this study were lost due to system failures or operator errors. This success may be a result of the relatively simple experimental design; nevertheless, we expect, given studies of equal complexity, that fewer experimenter errors would occur with our system compared to previous systems.

The difficulty found in establishing baselines contraindicates the use of particularly striking patterns, even though they consist of the intensity of colors of the stimulus image. Our mosaics produced dilation that indicated that subjects found them inherently interesting. The use of the 2-sec point as baseline may be inappropriate on the basis of Beatty's (1986) findings that pupil responses occur at about 300-500 msec after the onset of stimuli. On the other hand, including the influence of the mosaic could make the results more difficult to interpret. In future studies, we expect that a random pixel pattern made of the colors of the image or a neutral gray interstitial image will prove adequate for establishing baselines.
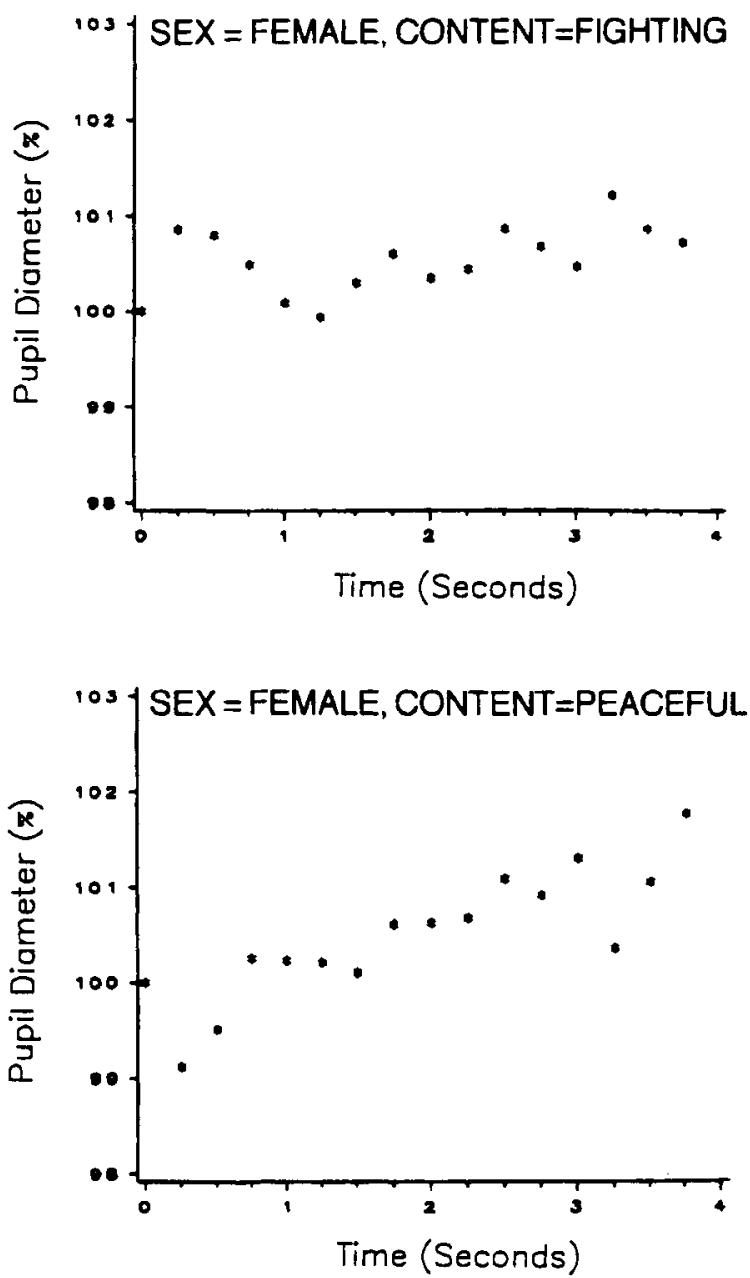

Figure 2. Pupil diameter (percent of baseline) over time for male and female subjects watching peaceful and fighting pictures. 

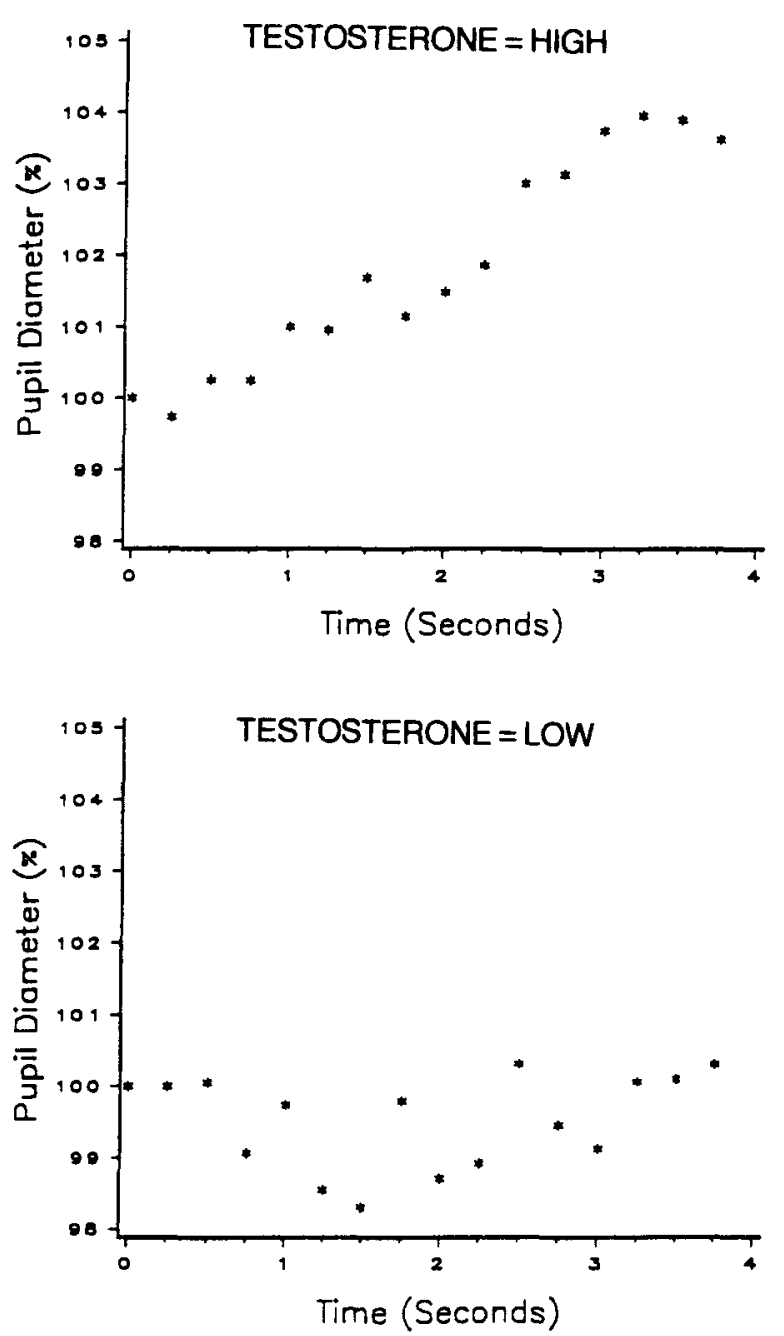

Figure 3. Pupil diameter (percent of baseline) over time for high- and low-testosterone males watching all pictures.

\section{GENERAL DISCUSSION}

The experimental setup is manageable and produces useful results. Pupillometry allows continuous monitoring of processing load over time. The high-fidelity audio and video color displays are well suited to studying reactions to complex stimuli, including faces and other objects, as well as multimedia shows. The simplicity of operation makes the system especially useful in getting consistent results from different subjects and different experimenters. The short time needed to run each subject means that large numbers of subjects can be run in a few weeks, making it possible to accumulate the sample sizes necessary to study individual difference variables in cognitive processing.

Collecting data with a desktop computer and passing it on to a mainframe computer for statistical analysis makes it feasible to handle large amounts of data and complex statistical designs, including designs with hundreds of levels of repeated measures factors. The advantage of ana- lyzing many repeated measurements is that it allows one to examine the profile of the pupil response over time, rather than being limited to examining latencies or peak responses within a given interval.

Recent advances in the operating system for the Macintosh allow for more precise and flexible control of timing of stimuli and data collection than has been heretofore available. Although the control in the demonstration study was adequate, the new "Time Manager" commands (operating system commands that allow more precise timing of program execution) offer greater precision and flexibility for more complex stimuli presentations.

The system is capable of using stimulus sets besides the video stimuli used in the demonstration study. Several studies conducted in our laboratory similarly confirm the utility of the system for audio stimuli. We are currently developing programs to show movies on the computer monitor while monitoring pupil size. The "Quicktime" data structure (a data structure that digitally codes movies and sounds into a compressed and manipulable format) allows digitized movies to be resources for programs and manipulated like other Pascal data types. Movies can be obtained from video camera or tape recorder and edited using one of several software packages. The stimuli in movies tend to seem natural, adding a new dimension to studying both cognitive and affective processes.

We have applied this system for use in social psychological study, but it might clearly be used in other areas of psychology. For instance, clinical psychological questions regarding the processing of negative information by depressed persons might be addressed using this system. Similarly, questions in developmental psychology regarding changes in information processing over the life span might be addressed.

This system is useful, adaptable, and simple to operate. For the researcher beginning research using pupillometry, this system has particular value because of the relative ease of initial setup. Modifying the programs to accommodate changing experimental demands has typically involved fairly small changes, requiring less than a day's work by a journeyman Pascal programmer. Alternative programming languages that might work equally well in the Macintosh environment include HyperCard and the $\mathrm{C}$ language. Although the studies described here have only tracked pupil diameter, information on direction of gaze is available from the ISCAN equipment, making it possible to examine what the subject was looking at in the moments prior to or during a change in pupil size. With the addition of other transducers to the setup, the A/D card could also be used to acquire data on skin resistance, heart rate, blood pressure, observer ratings, or other information to add to each line of the subject's data file. An experimenter needs little training or technical sophistication to produce consistent results when using our system.

\section{REFERENCES}

ARCHER, J. (1991). The influence of testosterone on human aggression. British Journal of Psychology, 82, 1-28. 
BEATTY, J. (1982). Task-evoked pupillary responses, processing load, and the structure of processing resources. Psychological Bulletin, 91 , 276-292.

BeatTy, J. (1986). The pupillary system. In M. G. H. Coles, E. Donchin, \& S. W. Porges (Eds.), Psychophysiology: Systems, processes, and applications (pp. 43-50). New York: Guilford Press.

BEATTY, J., \& WAGONER, B. L. (1978). Pupilliometric signs of brain activation vary with level of cognitive processing. Science, 199, 1216-1218.

Bernhardt, P. C., Cummings, A. C., DabBs, J. M., Riad, J. K., Hopper, C. H., \& Clay, J. K. (1992, August). Pupillometry and information processing in social psychology. Paper presented at the annual meeting of the American Psychological Association, Washington, DC

Blumenthal, T. D., \& CoOPER, J. A. (1990). Stimulus control and response measurement in human psychophysiological research using the Macintosh computer. Psychophysiology, 27, 479-480.

DABBS, J. M., JR. (1990). Salivary testosterone measurements: Reliability across hours, days, and weeks. Physiology \& Behavior, 48, 83-86.

DABBS, J. M., JR. (1991). Salivary testosterone measurements: Collecting, storing, and mailing saliva samples. Physiology \& Behavior, 49, 815-817.

Frey, L. A., White, K. P., \& Hutchinson, T. E. (1990). Eye-gaze word processing. IEEE Transactions on Systems, Man, \& Cybernetics, 20, 944-950.
HufF, C., \& SobiLoff, B. (1993). MacPsych: An electronic discussion list and archive for psychology concerning the Macintosh computer. Behavior Research Methods, Instruments, \& Computers, 25, 60-64.

Hutchinson, T. E., White, K. P., Martin, W. N., Reichert, K. C., \& FREY, L. A. (1989). Human-computer interaction using eye-gaze input. IEEE Transactions on Systems, Man, \& Cybernetics, 19, 1527-1533.

McConkie, G. W., Scouten, C. W., Bryant, P. K., \& Wilson, J. (1988). A microcomputer-based software package for eye monitoring research. Behavior Research Methods, Instruments, \& Computers, 20, 142-149.

SAS InSTITUTE, INC. (1982). SAS user's guide. Cary, NC: Author. Tryon, W. W. (1975). Pupillometry: A survey of sources of variation. Psychophysiology, 12, 90-93.

\section{NOTES}

1. The program and documentation are avalable on the Internet in: macpsych@stolaf.edu. See Huff and Sobiloff (1993) for details on how to access information in that forum.

2. All degrees of freedom corrections are Huynh-Feldt corrections.

(Manuscript received March 29, 1994;

revision accepted for publication September 26, 1994.) 